\title{
Simulation of the Mechanical Characteristics of a Granular Matter Tested under Biaxial Loading
}

\author{
Z. W. Bi, ${ }^{a, b, c, 1}$ W. F. Huang, ${ }^{a}$ and G. X. Xiao ${ }^{a}$ \\ ${ }^{a}$ Resource and Environmental Engineering, Jiangxi University of Science and Technology, Ganzhou, \\ Jiangxi, China \\ b Mine Safety Technology Branch, Chinese Coal Research Institute, Beijing, China \\ c State Key Laboratory of Coal Mining and Clean Utilization, Beijing, China \\ ${ }^{1}$ bizongwei1976@163.com
}

The mechanical characteristics of granular matter tested under biaxial loading are studied by numerical simulation. The stress-strain process, volume strain, coordination number, and shear zone are analyzed. The results indicate that the shear zone develops along with the coordination number and volume strain. With an increase in strain, the shear zone is centralized gradually. Moreover, the force transfer in the granular matter is also discussed.

Keywords: granular matter, force chain, biaxial test simulations, micromechanical.

Introduction. The stress-strain response of granular materials shows an incrementally nonlinear behavior when they are subjected to external loading. The macromechanical behavior stems not only from phenomena occurring at the contact surface, but also from mesoscale arrangements such as fabric evolution and force chains [1].

Numerical simulations using the discrete element method (DEM) have become a valuable tool in the study on different mechanical phenomena occurring at the micro- and mesoscale in granular materials [2]. The simulation of two-dimensional biaxial compression test has been performed on a densely packed assembly of polydisperse circular disks. A contact model that accommodates normal motions is based on the Hook law, while the model with tangential motion combines the Hook law with the Coulomb law to study the friction. A more detailed description of the contact laws, specimen preparation, and simulation parameters are given in [3]. Particle radii are uniformly distributed, and gravitational effects are neglected.

To create some initial configuration, non-contacting particles are distributed randomly within a box when the confining stress of $2 \mathrm{MPa}$ is applied to all four walls of the box. The assembly is then allowed to settle to a state where the kinetic energy is negligible. This isotropic assembly is subjected to boundary driven biaxial compression. The assembly undergoes compression at a constant strain rate in the vertical direction, while allowed to expand in the horizontal direction under constant confining stress. Figure 1 shows the boundary conditions and initial configuration, and Table 1 provides the material properties and parameters used. The compression rate in the vertical direction is $0.001 \mathrm{~m} / \mathrm{s}$.

The stresses are calculated considering that action of stresses on each set of opposite walls, where stresses on each wall are calculated by dividing the total force acting on the wall by the corresponding specimen length. The axial deviatoric stress is defined as

$$
\sigma=\sigma_{a}-\sigma_{c}
$$

where $\sigma_{a}$ is the axial stress and $\sigma_{c}$ is the mean confining stress.

The strains in both the vertical direction and the horizontal direction are calculated using the Eq. (2): 


$$
\varepsilon=\frac{L-L_{0}}{\left(L+L_{0}\right) / 2},
$$

where $L$ is the specimen current length in the corresponding direction and $L_{0}$ is the specimen original length in that direction. The specimen current height and width are used in stress and strain calculations.

$\mathrm{T}$ a b 1 e 1

Granular Material Parameters

\begin{tabular}{|c|c|c|c|c|c|}
\hline $\begin{array}{c}\text { Length } \times \text { width } \\
(\mathrm{mm})\end{array}$ & $\begin{array}{c}d_{\max }, \\
\mathrm{mm}\end{array}$ & $\begin{array}{c}d_{\min }, \\
\mathrm{mm}\end{array}$ & $\begin{array}{c}\text { Friction } \\
\text { coefficient }\end{array}$ & $\begin{array}{c}\text { Contact } \\
\text { stiffness }(\mathrm{N})\end{array}$ & $\begin{array}{c}\text { Density } \\
\left(\mathrm{kg} / \mathrm{m}^{3}\right)\end{array}$ \\
\hline $120 \times 60$ & 6.3 & 4.6 & 0.5 & $5 \cdot 10^{8}$ & 1000 \\
\hline
\end{tabular}

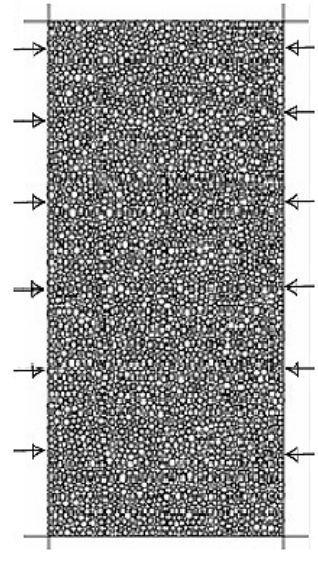

Fig. 1. Simulation model.

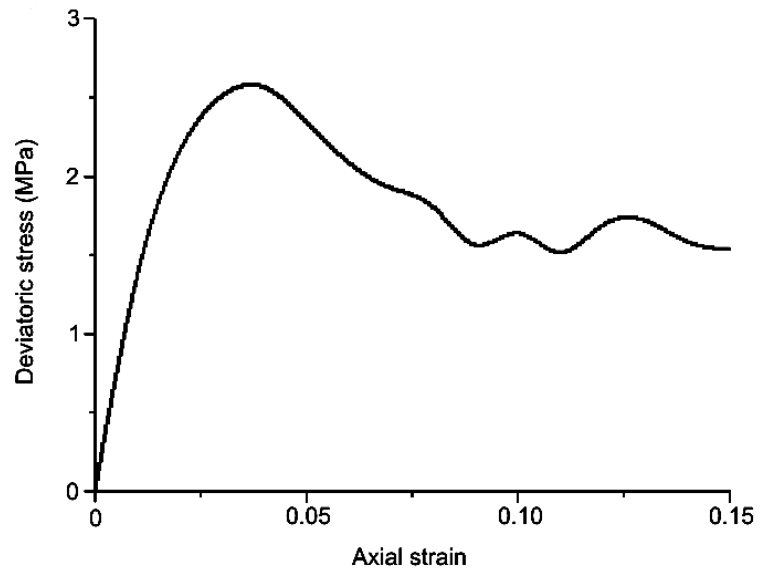

Fig. 2. Stress-strain curve.

The volumetric strain is calculated using the Eq. (3):

$$
\varepsilon_{V}=\varepsilon_{x}+\varepsilon_{y} .
$$

Macromechanical Observations. The bulk mechanical behavior of the virtual specimen is represented by stress-strain curve as shown in Fig. 2. The peak or maximum deviatoric stress attained using the virtual specimen is approximately $3 \mathrm{MPa}$ with the axial strain of approximately $2.5 \%$. Post peak behavior is observed as strain softening.

The strain evolution in the system exhibits distinct regimes of deformation, and the essential aspects of deformation at different stages during loading can be described as follows. Typically, dense assemblies of cohesive granular materials are compressed in a period of strain-hardening regime, followed by strain-softening and, finally, the so-called residual stress state [4-6]. The strain hardening regime is characterized by a monotonic increase with axial strain to a peak value. During the post-peak phase the deviatoric stress drops significantly along with progressive development of shear bands until it reaches a steady state value called the residual stress state. Relatively large fluctuations are observed at the post peak stage. The ensuing strain-softening regime is characterized by a sequence of precipitous drops. A single persistent shear band governs the dynamics in the residual stress state regime. 
Volumetric Strain. The total volumetric strain undergoes compression, dilation or increase of volume, and then continuing deformation, as it is shown in Fig. 3. The sudden decrease of dilation rate coincides with the onset of shear band. The maximum rate of dilation coincides with the peak strength.

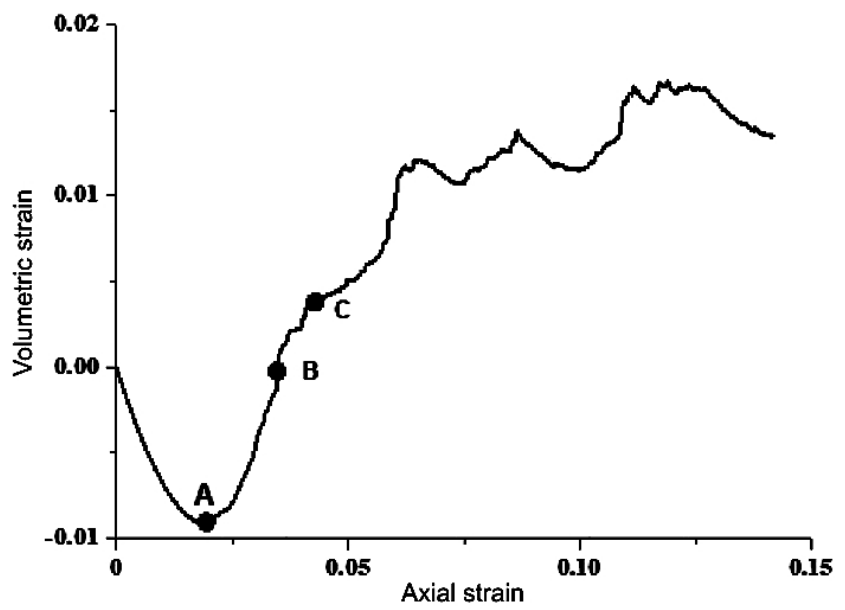

Fig. 3. Volumetric strain curve.

As it can be seen under compression the value $\varepsilon_{V}$ decreases to be negative. While the stress increases, the system displays elastic strain at this time. When $\varepsilon \approx 2 \%$, $\varepsilon_{V}=-0.8 \%$, the system moves towards the most dense state (point $A$ ) with the maximum volume fraction. When the value $\varepsilon>2 \%, \varepsilon_{V}$ starts to increase, the system is in the plastic stage. Until $\varepsilon=3.5 \%$ and $\varepsilon_{V}=0$, the volumetric strain returns to its initial value (point $B$ ). After $\varepsilon>3.5 \%$, the rate of change (slope) remains the same, even though there is slight fluctuation in the volumetric strain. When $\varepsilon=4.2 \%, \varepsilon_{V}$ continues to increase, but the rate is significantly lower (point $C$ ). Hereafter, the system reaches its critical state where the internal force chain collapses and re-combines constantly, and larger fluctuations of $\varepsilon_{V}$ appear, respectively.

Shear Bands. Some analysis has been conducted on the displacement of shear zone [7-12]. For instance, Vardoulakis and Aifantis [7] and Chambon et al. [8] studied the shear band based on the second gradient method. Meanwhile, Cundall and Strack [2], Bardet and Proubet [9] examined shear strain localization by using PFC discrete element software.

This paper is focused on the formation and development of shear band considering the rotation of particles. In Fig. 4, white spots represent the particles with rotation angle $\alpha \leq 5^{\circ}$, grey represents $5<\alpha<10^{\circ}$, and dark represents $\alpha \geq 10^{\circ}$. As it can be seen, in the final state with $\varepsilon=15 \%$, most particles with large rotation angle are found to be concentrated inside the shear band.

As it is shown in Fig. 4 the formation of shear bands is accompanied by particle rotation. When $\varepsilon=2.2 \%$, plastic deformation takes place in the system, which changes from the densest to loose. At this time, some small shear bands formed by the particles with larger rotation angles are observed, and the band width is similar in value to single particle diameter. It is indicated that localization occurs inside the system before the stress reaches the peak. These small shear bands are parallel to each other and extend along their own direction. When $\varepsilon$ increases, the shear bands start to combine and change their direction near the combination point. The shear band in one direction becomes more pronounced, while those in another direction start to disappear. In other words, the dominant shear zone starts to form. With an increase of $\varepsilon$ the multiple shear band formation, combination, and 


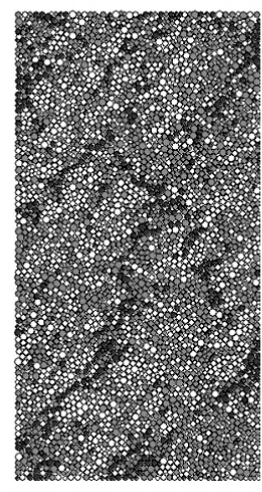

a

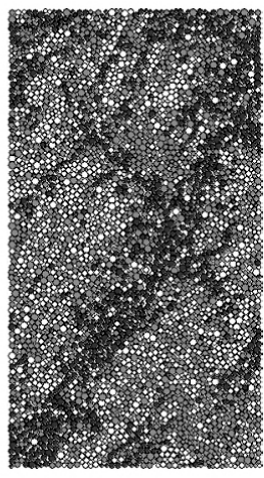

$\mathrm{b}$

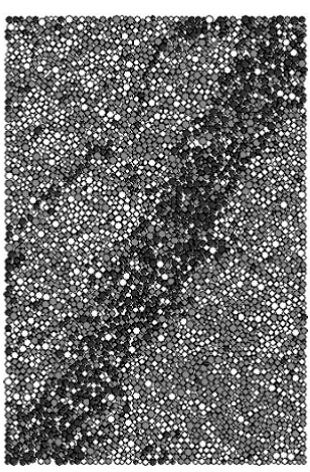

C

Fig. 4. Distribution of particle rotation angle: (a) $\varepsilon=2.2 \%$; (b) $\varepsilon=4 \%$; (c) $\varepsilon=15 \%$.

bifurcation can be observed. At $\varepsilon=4 \%$, more particles have larger rotation angle, and the distribution is concentrated. A distinct shear zone of certain thickness crosses the entire system indicating the system critical state. With further increase of $\varepsilon$ the shear band forms eventually, whose width is of about 6-8 particle diameter. Meanwhile, small shear bands are observed to disappear. The critical state and the direction of shear band indicate that the granular system is consistent with the Mohr-Coulomb standards. Similar phenomenon is also observed during testing on dry sand using scanning electron microscope, digital optical image analyzer, and X-ray equipment.

Coordination Number. The simplest way to study the microstructural arrangement of granular sample is to examine the evolution of coordination number $Z$. The coordination number $Z$ is defined as mean number of contacts per particle. To observe the change of $Z$, three measurement balls are placed in the system, as it is shown in Fig. 5a. The evolution of coordination number is shown in Fig. 5 b.

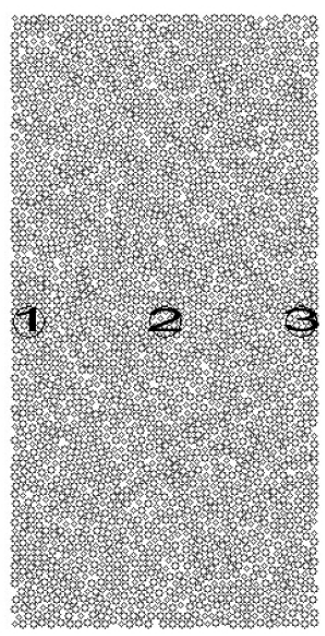

a

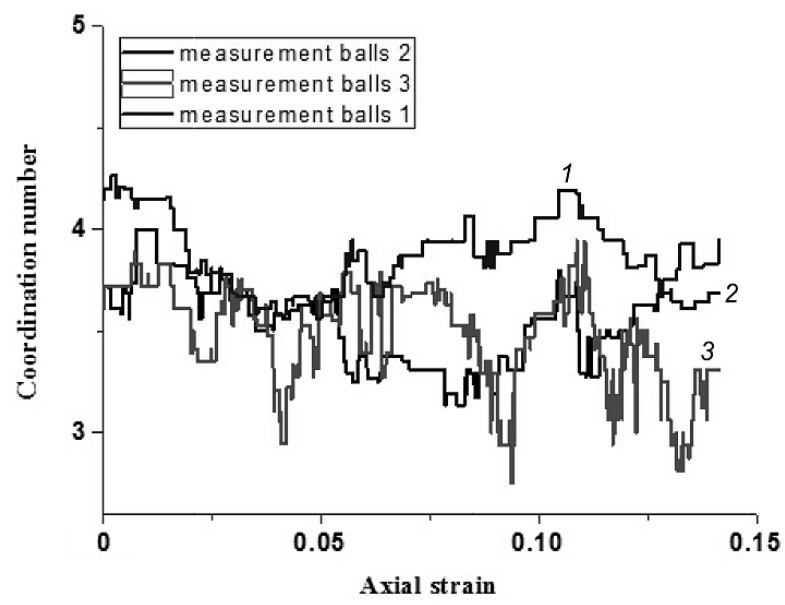

b

Fig. 5. Positions of three measurement balls (a) and evolution of coordination number (b).

At low strain values there is an insignificant increase of the coordination number with the increase of strain. And the amount of increase is different with various measurement 
balls. When the strain is $1 \%$ the coordination number of ball 2 shows a peak value of 3.8 , then it decreases. Meanwhile, the coordination numbers of balls 1 and 3 remain at 4.4 and 4.0 , respectively. When the strain is $2.8 \%$, all particles are without contact, and the system is at the strain softening stage. When the strain is $4.5 \%$, the system has reached the residual stress state. The coordination numbers of balls 1 and 3 are observed to change only within a small range, which indicates that the system has completed the transfer from $\mathrm{X}$ shear zone to the sole shear zone.

Development of Force Chain. Figure 6 shows the development of force chain at different stages. At $\varepsilon=0$, the granular matter is in isotropic compression state. A ring-like structure is observed in most of force chains, their spatial distribution is relatively uniform (Fig. 6a). There is no obvious inhomogeneity of force chain intensity, which means the load at the boundary/bounds is distributed evenly on most particles.

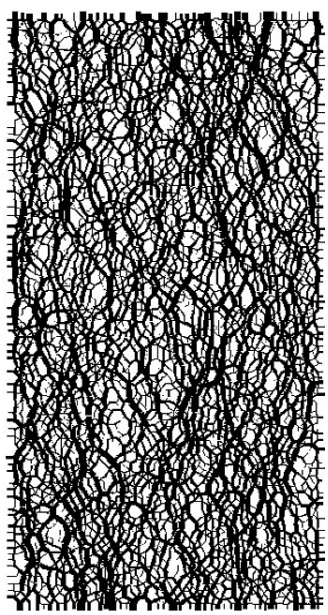

a

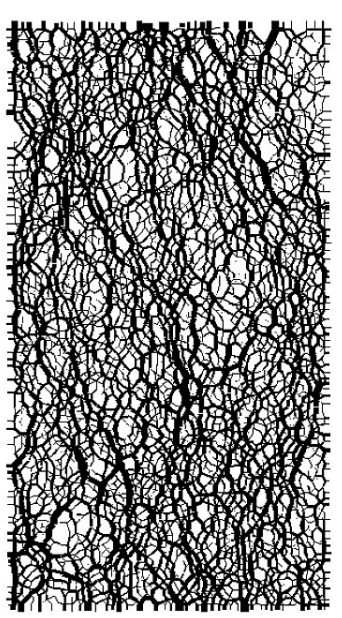

b

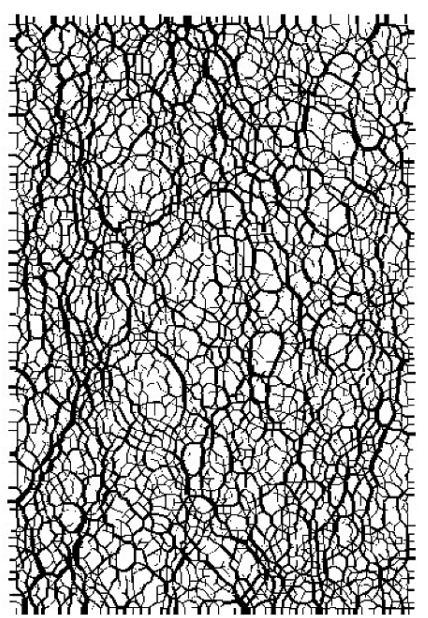

C

Fig. 6. Development of column-like structure and its fracture in shear bands (scale $=3 \cdot 10^{6}$ ).

If the load is imposed on the upper and lower boundaries, the major direction of principal stress is vertical, while the minor is horizontal. The force chain structure is changed to column parallel to the vertical direction of major principal stress (Fig. 6b). At this time, the intensity of force chain shows some inhomogeneity. The strong force chains bear the load in the vertical direction. Although the weak ones do not share the vertical load, they play an important role in supporting the vertical force chains and preventing them from bending.

When the system experiences the stress dilatancy phase with $\varepsilon=2.8 \%$, the local void ratio increases, and the force chains become annular (or spiral) and form the so-called arch. Due to reduction of contact number, the strong force chains lose the support of weak force chains, and the particles rotate significantly. The annular force chain is very unstable and tends to fracture resulting in a whirlpool in the local area. When the force chain is broken, microcracks appear. The development of multiple microcracks leads to the formation of shear bands. With the dominant shear band the force chains outside the shear band remain to be a column structure parallel to the vertical direction, while the ones inside the shear band undergo significant bending. It is indicated that the principle stress inside the shear band has rotated (Fig. 6c). The bent force chains inside the shear band are ring-like, and their width is consistent with the width of shear band. After the formation of shear band, the direction of particle contact angle also changes, since some force chains inside the shear band are not parallel to the principal stress, however, are at certain angle. 
Conclusions. In this paper, the relation between the inter-particle factors and the macromechanical behavior of granular materials was investigated. The results show that the variation of stress has a significant effect on the evolution of displacement, coordination number and force chain in the granular matter. With an increase in strain, the shear zone becomes more evident. An overall translation phenomenon is observed when the strain is over $4.8 \%$. Furthermore, it is found that the system at residual stress state develops force chains that are highly susceptible to fracture, which leads to strong stress fluctuations. Stress collapses also reduce the contacts, and therefore result in a temporary stability of the granular specimen.

Acknowledgments. This work has been supported by Chinese Postdoctoral Science Foundation (2014M550358).

1. F. Darve, "The expression of rheological laws in incremental form and the main classes of constitutive equations," in: F. Darve (Ed.), Fractured and Jointed Rock Masses, Taylor \& Francis, London (1990), pp. 123-148.

2. P. A. Cundall and O. D. L. Strack, "A discrete numerical model for granular assemblies," Geotechnique, 29, No. 1, 47-65 (1979).

3. Q. C. Sun and F. Jin, "Force chain in a uniaxially compressed static granular matter in 2D," Acta Phys. Sinica, 58, No. 9, 6549-6553 (2009).

4. A. A. Pena and A. F. Lizcano, "Biaxial test simulations using a packing of polygonal particles," Int. J. Num. Anal. Meth. Geomech., 32, No. 2, 143-160 (2008).

5. A. Tordesillas, "Force chain buckling, unjamming transitions and shear banding in dense granular assemblies," Phil. Mag., 87, No. 32, 4987-5016 (2010).

6. S. Luding, M. Latzel, W. Volk, et al., "Discrete element simulations to a continuum model," Comp. Meth. Appl. Mech. Eng., 191, No. 1, 21-28 (2001).

7. I. Vardoulakis and E. C. Aifantis, "A gradient flow theory of plasticity for granular materials," Acta Mech., 87, No. 3, 197-217 (1991).

8. G. Chambon, J. Schmittbuhl, A. Corfdir, et al., "Shear with comminution of a granular material: microscopic deformations outside the shear band," Phys. Rev. E, 68, 011304 (2003).

9. J. P. Bardet and J. Proubet, "Numerical simulations of shear bands in idealized granular materials," Solid State Phenom., 23-24, No. 1, 473-481 (1992).

10. J. Desrues and G. Viggiani, "Strain localization in sand: an overview of the experimental results obtained in Grenoble using stereophotogrammetry," Int. J. Num. Anal. Meth. Geomech., 28, No. 4, 279-321 (2004).

11. W. J. Wang, X. Z. Kong, and Z. G. Zhu, "Friction and relative energy dissipation in sheared granular materials," Phys. Rev. E, 75, 041302 (2007).

12. M. Oda, K. Iwashita, and H. Kazama, "Micro-structure developed in shear bands of dense granular soils and its computer simulation - mechanism of dilatancy and failure," in: N. A Fleck and A. C. E. Cocks (Eds.), IUTAM Symp. on Mechanics of Granular and Porous Materials, Kluwer, Dordrecht (1997), pp. 353-364. 